\title{
Internal Application of Ultra-Fast Temperature Sensitive Paint to Hydrogen Combustion Flow
}

\author{
Jan Martinez Schramm $\left.{ }^{(}\right)$and Leni Schmidt \\ Department Spacecraft, German Aerospace Center, Institute for Aerodynamics and \\ Flow Technology, Bunsenstr. 10, 37073 Göttingen, Germany \\ $\{$ Jan. Martinez, Leni.Schmidt\}@dlr.de
}

\begin{abstract}
The paper will discuss an experimental approach to determine wall surface heat flux in a hydrogen combustion chamber using a temperature sensitive paint. This measurement technique represents a non-intrusive method to determine wall surface heat flux with a high spatial resolution. The application of this method to an internal flow, generated in a hydrogen fueled combustion wind tunnel model, is a new approach in the application of the measurement technique. The wind tunnel model represents the small scaled flight experiment configuration of the MR2 vehicle of the EU co-funded Long-Term Advanced Propulsion Concepts and Technologies project. The paper will discuss the special demands and prerequisites needed to apply TSP in high temperature flows. The experimental setup will be described and the feasibility of the application of TSP to internal flows and the resulting technical demands will be reviewed. The obtained result will be discussed with respect to the validity by comparison to a numerical simulation of the combustor flow.
\end{abstract}

Keywords: High enthalpy $\cdot$ Shock tube $\cdot$ Heat flux $\cdot$ Internal flow $\cdot$ High temperature hypersonic flow $\cdot$ Hydrogen combustion $\cdot$ HEG $\cdot$ TSP $\cdot$ SSFE $\cdot$ LAPCAT

\section{Introduction}

The precise determination of the surface heat flux is mandatory for experiments in high enthalpy impulse facilities [1]. The usage of surface-mounted temperature sensors like thermocouples or thin film gauges is common in these facilities. The High Enthalpy Shock Tunnel Göttingen, HEG, is one of the major European hypersonic test facilities of this kind [2]. Thermocouples and thin film gauges guarantee fast response times in the order of a few microseconds and therefore a high temporal resolution. But the spatial resolution is limited and can only be increased by increasing the number of sensors installed in a wind tunnel model. Additionally, it is challenging to attach sensors on complex or sharp-edged models. A non-intrusive option is the use of the temperature-sensitive paint technique

(C) The Author(s), under exclusive license to Springer Nature Switzerland AG 2021

A. Dillmann et al. (Eds.): STAB/DGLR Symposium 2020, NNFM 151, pp. 121-131, 2021.

https://doi.org/10.1007/978-3-030-79561-0_12 
(TSP). The application of TSP enables the measurement of spatially resolved surface temperatures which allow for the subsequent calculation of surface heat fluxes. The application of the technique to an internal flow will be presented in this paper. The measurement technique will be used to determine wall surface heat flux in the propulsion unit of the small scale flight experiment configuration (SSFE) of the Long-Term Advanced Propulsion Concepts and Technologies (LAPCAT) MR2 vehicle [3] in order to validate the numerical modeling of the flow $[4,5]$. TSP has been successfully applied in HEG [6] and to the external flow in the SSFE intake [7].

\section{Theoretical Background}

A TSP typically consists of a polymer binder, luminescent molecules called luminophores and a solvent. Here, the used TSP was a mixture of Dichlorotris(1, 10-phenanthroline) ruthenium $(\mathrm{Ru}(\mathrm{phen}))$ serving as luminophore dissolved in the polyamide AQ Nylon (P-70) and ethanol. The binder has to be oxygen impermeable in chemically reacting hypersonic flows to prevent recombination processes in the boundary layer and on the surface. In addition, the impermeability is mandatory to prevent any pressure dependence of the TSP. Figure 1a shows a schematic of a typical TSP setup. The paint is illuminated by light emitting diodes (LEDs) having a specific wavelength $\lambda_{e x}$ in order to excite the luminophores of the paint, while a light sensitive device detects the luminescence signals emitted at a wavelength $\lambda_{e m}$ from the TSP. The luminophores are exited to higher electronic states by the absorption of photons. The return to the ground state occurs through different mechanisms, either non-radiative relaxations or radiative decay [8]. One of those is the deactivation by the emission of a photon (photoluminescence). The other predominant mechanism is the thermal quenching which is a radiation-less process due to particle collisions. This process is temperature dependent, which means a rise in temperature increases the probability of radiation-less transitions. As a result, there are less emissions and thus the luminescence of the paint decreases. The energy loss due to the radiation-less transitions causes a shift in the wavelength. As shown in Fig. 1b, the spectrum of the excitation light source (LED) differs from the detected emission spectrum of the TSP. The maximum in wavelength of the emitted luminescence radiation is increased in comparison to the excitation wavelength. The difference in wavelength between both maxima is defined as the Stokes shift. A large Stokes shift is advantageous, because it facilitates the mandatory separation of luminescent emission from excitation radiation before the detection. For this purpose, a filter is placed in front of the detector to suppress light of shorter and longer wavelengths. For the application in hypersonic flows and under ultra-fast conditions, there are some special requirements for the paint due to the limited test time [9]. As already discussed, a temperature increase in the TSP leads to a lower intensity of the emitted light. This means the detectable temperature rise is limited; the signal becomes zero at an upper temperature limit. The temperature sensitivity of the TSP has to be adapted to the measurement situation. 

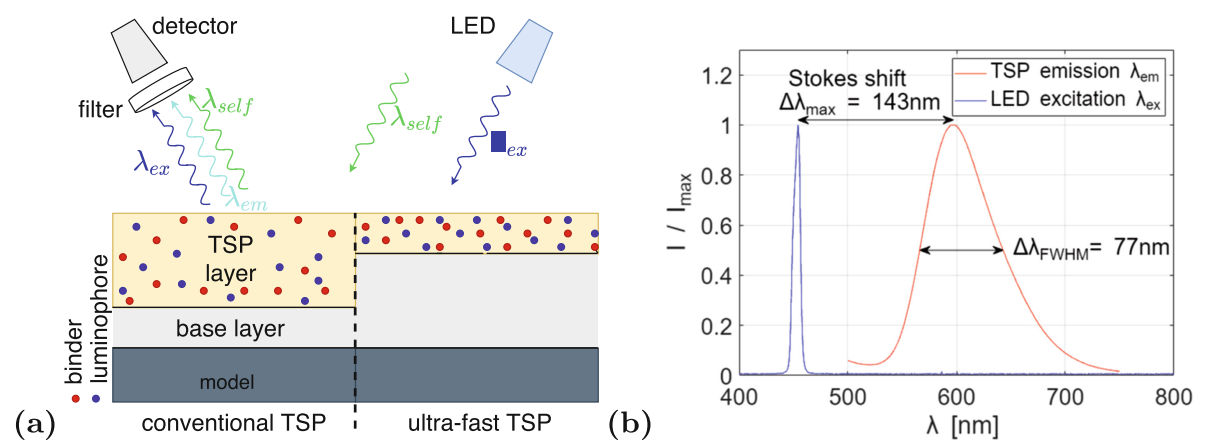

Fig. 1. (a): Schematic of typical and high-speed TSP set-up. (b): Emission and excitation spectrum of the selected TSP under excitation with blue LED $\left(\lambda_{e x}=460 \mathrm{~nm}\right)$.

Further, the luminescent lifetime of the luminophore of the TSP has to be significantly shorter than the test time and thus, in our case, it is in the order of less than 1 microsecond. When the TSP method is used in short duration flows, the detection instrument needs to be set up for short exposure times to resolve the fast changes appropriately. But these short exposure times require a fast response and high intensity luminescence signals of the paint [9]. The utilisation of a reflective base layer helps to increase the intensity of the luminescence signal and is essential in our case. Normally, a thick TSP layer causes an increase of the signal. But the paint's response time is proportional to the square of the paint thickness, so a rather thin layer in order of a few micrometers is required for the application in ultra-fast context [8]. As illustrated in Fig. 1a, the TSP layer for ultra-fast TSP is much thinner than the base layer in comparison to a conventional TSP setup. If long time scales are considered, temperature equilibrium between TSP and base layer can be assumed and the luminescence can directly be related to the temperature of both, the TSP and the base layer. In ultrafast applications, the TSP layer needs to be thin enough to assume a negligible effect of it on the heat conduction process. The heat conduction process has to be considered with respect to time. To do so, we can treat the base layer as a uniform semi-infinite medium; this allows to follow to the analysis for thin-film gauges and other conventional sensors, as described by Schultz and Jones [10]. Under these presuppositions, it is possible to recover the heat transfer $\dot{q}_{s}$ from time dependent temperature profile in a discrete form [11]:

$$
\dot{q}_{s}\left(t_{n}\right)=2 \frac{\sqrt{\rho c k}}{\pi} \sum_{i=1}^{n} \frac{T\left(t_{i}\right)-T\left(t_{i-1}\right)}{\sqrt{t_{n}-t_{i}}+\sqrt{t_{n}-t_{i-1}}} .
$$

$T\left(t_{i}\right)$ is the surface temperature obtained at time $t_{i}$. The heat flux depends on the thermal properties $\sqrt{\rho c k}$ with the density $\rho$, specific heat capacity $c$ and the heat conductivity $k$ of the base layer material. For the special case of a constant heat flux, the surface temperature can be expressed as [10]: 


$$
T(t)=\frac{2 \dot{q}_{s}}{\sqrt{\pi}} \sqrt{\frac{t}{\rho c k}} .
$$

Additionally, the implementation of the TSP technique in high enthalpy test facilities like the HEG creates some other challenges due to the special test conditions [12]. In hypersonic high speed flows, a self-illumination of the test gas may occur because of the arising high temperatures, this leads to a superimposition with the signals from the TSP luminescence.

\section{Experimental Apparatus and Data Processing}

The layout of the hydrogen-fueled vehicle is characterized by an aerodynamically ecient wave rider design and the integration of a propulsion unit [13]. A schematic of the 1.5-m-long SSFE configuration installed in the HEG test section is shown in Fig. 2a. The model is held by two feet that are fixed on the test section rail system. The vehicle's inward turning intake was designed based on a modied Busemann template ow eld and has an elliptical capture area [13]. The intake leads to an elliptical combustion chamber in which a two-stage multi-strut injector system is installed. Directly behind the intake, an upstream stage consisting of two semi-struts is injecting the hydrogen almost normal to the flow while downstream a full strut injects in the direction of the flow from a central position. The nozzle merges the elliptical combustor cross section into a circular shape and then expands. The hydrogen is supplied through a valve into a pipe that attaches to the model and supplies two steel bottles, one for each injector. Once the system is filled to the required pressure, the valve is closed and the hydrogen is injected into the combustor a few milliseconds before the run. The model is instrumented with 44 pressure transducers. The transducers in the combustor are located at the sidewall, while more transducers cover the region near the intake and in the nozzle. Their transducer signals are amplified

(a)

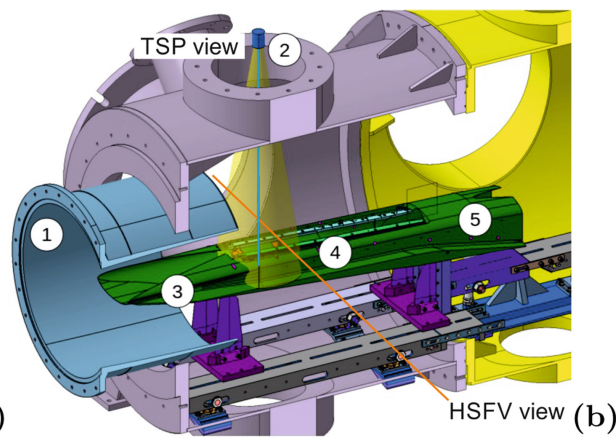

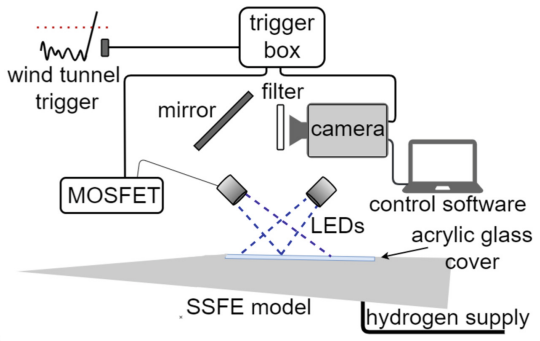

(b)

Fig. 2. (a) Schematic of the model installation in the HEG test section. 1: HEG nozzle; 2: LED; 3: intake; 4: combustor; 5: thrust nozzle. (b) Schematic of the TSP setup. 
to increase the signals before they are recorded by the data acquisition system. A high speed camera is used in conjunction within a Schlieren setup to visualize the flow through the optical windows perpendicular to the flow direction. The high speed flow visualisation (HSFV) system allows to visualize the outer flow, ensure proper flow start up and can indicate unwanted movement of the model. A transient recorder is used for data acquisition of the wind tunnel instrumentation, the model instrumentation and all relevant signals of the TSP and Schlieren system. For the TSP measurements a top view has been realized. A schematic of the TSP setup is shown in Fig. 2b. Ten LEDs with each emitting at a wavelength of $460 \mathrm{~nm}$ illuminate the combustion chamber wall through the upper optical window of the shock tube. They are aligned in such a way that an optimal homogeneous illumination of the combustion chamber wall is attained. To optimize the power output of the LEDs, the currents used have to be maximized. This can only be achieved for short amounts of time. Therefore, the LEDs are loaded with high currents using a metal oxide semiconductor field-effect transistor (MOSFET) for approximately $8 \mathrm{~ms}$. We use $I=64.7 \mathrm{~A}$ and $U=51 \mathrm{~V}$ to drive this pulse, which results in a total power of $3299.7 \mathrm{~W}$. The complete acquisition chain is triggered by the tunnel signals. Once the trigger is active, the high-speed high-resolution uesd to visualize the TSP starts the recording. The camera records at a frame rate of $60 \mathrm{kHz}$ and an exposure time of $2.5 \mathrm{\mu s}$. The image of the combustion chamber wall is mapped onto the camera chip through a mirror and a lens system. The inclination of the mirror can be changed easily to obtain an optimal image view. To allow optical access to the internal flow, the combustion chamber is closed with an acrylic glass cover. This causes an optical distortion in the image between the view without (Fig. 3a) and with cover (Fig. 3b). Due to that, the measurements have to be corrected. Therefore, points in a grid are marked on the layer which enable the comparison of the view with and without the cover. White light recordings of the view with and without the cover have been done to make the determination of the distortion possible. Moreover, a coordinate measuring arm was used. This device has a special needle tip. This tip can be pointed to each grid marker and stores the 3D coordinates in relation to a defined coordinate system. The acquired data set of the grid can be used later to build a 3D model of the combustion chamber and thus reconstruct the $3 \mathrm{D}$ internal heat flux from the TSP recording. The operating condition used in HEG for the experimental investigation was condition XIII [2]. To transfer the measured TSP intensities and obtain results to compare to the numerical modeling, the data need to be processed. The first step is to normalize the acquired intensity data. This correction can be achieved by taking the ratio $I / I_{0}$ of each shot image to the corresponding reference image for each pixel. As described above, an optical distortion occurs caused by the acrylic glass cover. Therefore, points in a grid were marked on the foil before the test to identify the distortion. As illustrated in Fig. 3c, the points are particularly distorted along the $\mathrm{y}$-direction, while the displacement in $\mathrm{x}$-direction is rather negligible small. The DLR software ToPas enables the warping of a picture. It compares the grid markers on the images with and without cover and creates a projection matrix 

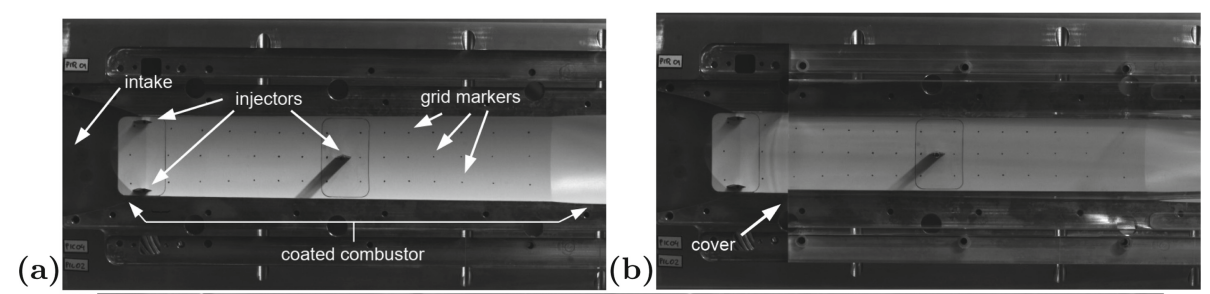

(c)

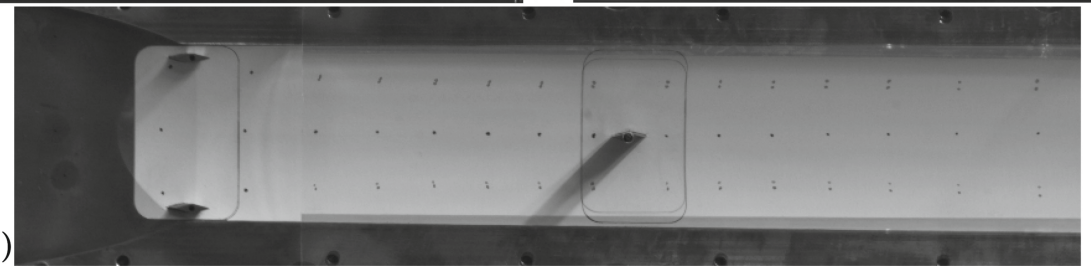

Fig. 3. Before the experiment white light shots were used to compare the distortions caused by the cover. a) without cover. b) with cover. c) overlay of both images.

between the grid markers. The area around the grid is interpolated. With the help of this projection matrix, each distorted image can now be corrected. The TSP technique is based only on the reduction of the luminescence intensity. But the hydrogen burning causes a self-illumination of the flow and thus leads to an increase in intensity. In order to separate the TSP signals, a mask was overlaid for pixels whose intensity deviates upwards more than $8 \%$ from a defined reference image at the beginning. This reference frame was chosen to the point when the LED excitation stabilized and thus initial fluctuations in the intensity stopped. The results are smoothed using a moving average filter to minimize the noise signals. Next, the intensity values need to be transferred to temperatures. Using the time dependent temperatures, the heat flux can be determined using Eq. 1.

\section{Results}

We will discuss and present the data we have been able to obtain from one experiment, being performed as a feasibility study. The measurement technique itself, even though it is quite simply from theory and can be described in a straight forward manner, is quite complex when real experimental data has to be analyzed. The first thing to note here is that the outer regions at the walls of the combustion chamber have to be excluded from the analysis. The reason for that is the extreme gradient of distortion caused by the strong bending of the cover in that area. We excluded this region in the analysis. Figure 4a shows a pressure recording of a sensor in the combustion chamber as a blue line and the pressure which is recorded in the reservoir of the hydrogen combustion unit as a red line. Please note that the units for the hydrogen reservoir pressure are given in arbitrary units. The time axis is normalized to the instant in time, when 
(a)
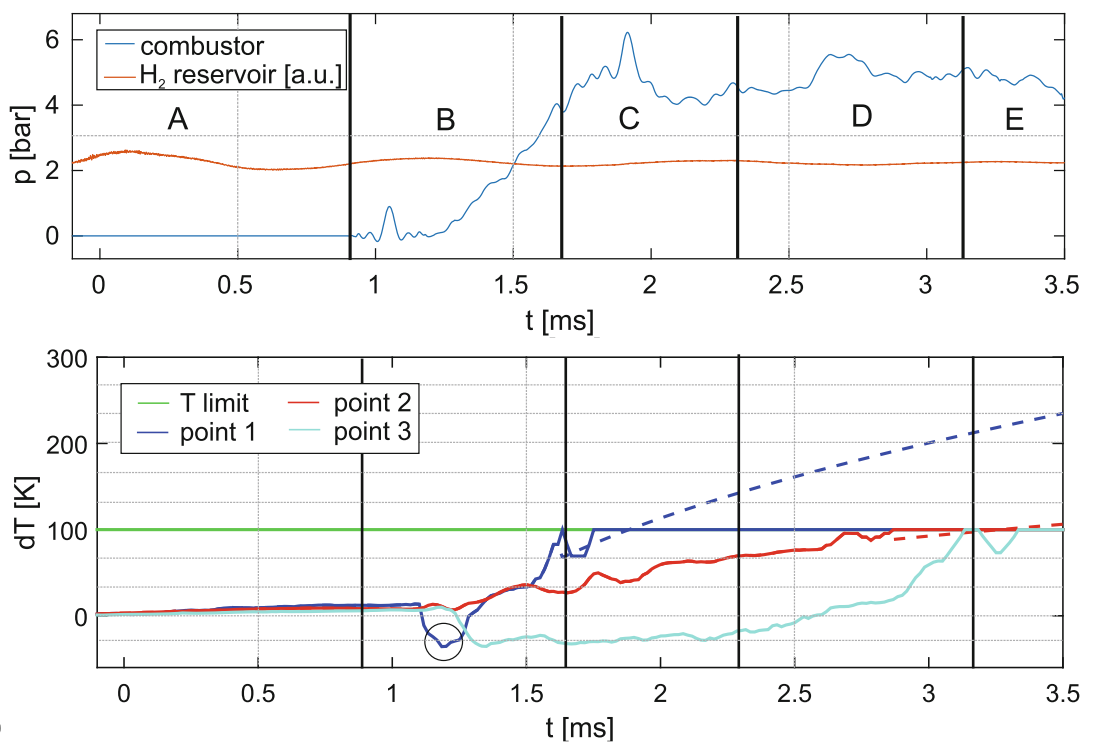

(b)

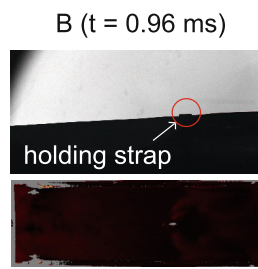

(c)

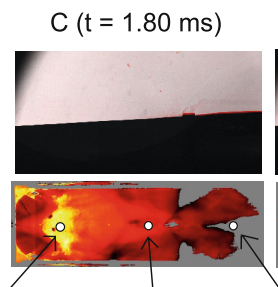

$\mathrm{D}(\mathrm{t}=2.43 \mathrm{~ms})$

$E(t=3.35 \mathrm{~ms})$
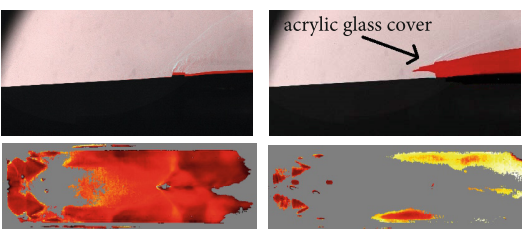

point 1

point 2

point 3

Fig. 4. a) Pressure trace of sensor in the combustion chamber and pressure in hydrogen reservoir. b) Time-dependent temperature profile for three points with extrapolations. c) Selected TSP heat flux distributions and false-color HSFV images (acrylic glass cover in red).

the reservoir of the hypersonic nozzle of HEG starts $(t=0 \mathrm{~ms})$. The flow needs roughly $1 \mathrm{~ms}$ before it reaches the SSFE model. The time needed to obtain a constant mass flow rate through the injectors is larger than the flow start time in the combustion chamber, therefore it is mandatory to start the hydrogen injection process prior to the flow start. This process can be seen in Fig. 4a. The injection process is already steady at roughly $0.7 \mathrm{~ms}$ before the arrival of the test gas air in the combustion chamber, which happens at $1 \mathrm{~ms}$. To discuss the evaluation process, we divide the experiment in five phases denoted by the letters A through $\mathrm{E}$ as given in Fig. 4a. Phase A is the observation phase prior to the actual experiment and before the flow start in the combustor. During phase $\mathrm{A}$, the hydrogen is floating into the combustion chamber and a small increase in surface wall temperature is notable already in the time interval $t=0-0.7 \mathrm{~ms}$ as 
given in Fig. 4b. This data shown in Fig. 4b originate from three specific points (1), (2) and (3) on the TSP layer and the corresponding locations are shown in Fig. 4c. The first point located just behind the axial position of the two semi struts and in the symmetry plane shows the temperature development in a high temperature region, which can also be identified in the heat flux distributions given in Fig. 4c. We can observe from the temperature development with respect to time, that the measurement range for most of the points in the combustor's front part is exceeded at approximately $t=2.43 \mathrm{~ms}$ (the limit is marked with the green line). Some individual points like point (1) exceed the limit temperature already before at about $t=1.8-1.9 \mathrm{~ms}$. After this time $t=2.43 \mathrm{~ms}$, the heat flux integration using Eq. 1 is not valid anymore and the evaluation must stop. The gray areas in Fig. 4c mark the regions, where the heat flux determination had to be stopped due the above described effect. However to investigate this region, the assumption of a constant wall surface heat flux is made and an extrapolation of the time behaviour based on Eq. 2 can be performed. The extrapolation of the data is given by the dotted blue line in Fig. 4b. A second effect that has to be considered in the data is the illumination of parts of the CCD chip which originates from the hydrogen combustion during the startup and could not be suppressed for the run. This disturbing light which will increase the intensity on the chip will force the evaluation into a negative temperature and therefore a negative heat flux reconstruction. Regions with this behaviour are completely masked out in the evaluation and are given as grey regions in Fig. 4c. The effect can be seen in the signal around $t=1.2 \mathrm{~ms}$ and is marked with a black circle. The overshot in intensity will lead to a negative temperature undershoot in the evaluation, this has to be considered when extrapolating the data. A lot of TSP readings fall in phase $\mathrm{B}$, which covers the time during the flow start up in the combustor. The Schlieren images given in Fig. 4c (top row) allow to define a third phase, which we denote with phase C. The acrylic glass insert forming to top wall of the combustor was not able to withstand the large forces at some point in time and started to bend, this can be observed in the Schlieren images in Fig. 4c. The acrylic glass cover, separating from the model, has been colored in red for better visibility. At $t=2.43 \mathrm{~ms}$, the covers starts to deform slightly and this ends the phase $\mathrm{C}$ at which the experiment remains unchanged. The temperature development for the point (3) in Fig. 4b shows a negative development of the wall surface heating. There is no physical process possible that would allow for a negative wall surface heating as we observe it. The reason behind this behavior is the same as for the negative overshoot, we explained earlier: the camera sees intensities originating from the hydrogen combustion process during the experiment. The locations that show this behaviour can not be treated and are also marked in grey (see Fig. 4c). The phase D is not considered for the evaluation anymore, here the cover deforms already and it can not be assured that the flow will not be influenced. The phase E shows the phase when the cover lifts of the model. It is interesting to note that the pressure is not strongly influenced by this effect. To show the flow processes we can observe by the TSP measurement, the inter- and extrapolated temperature 

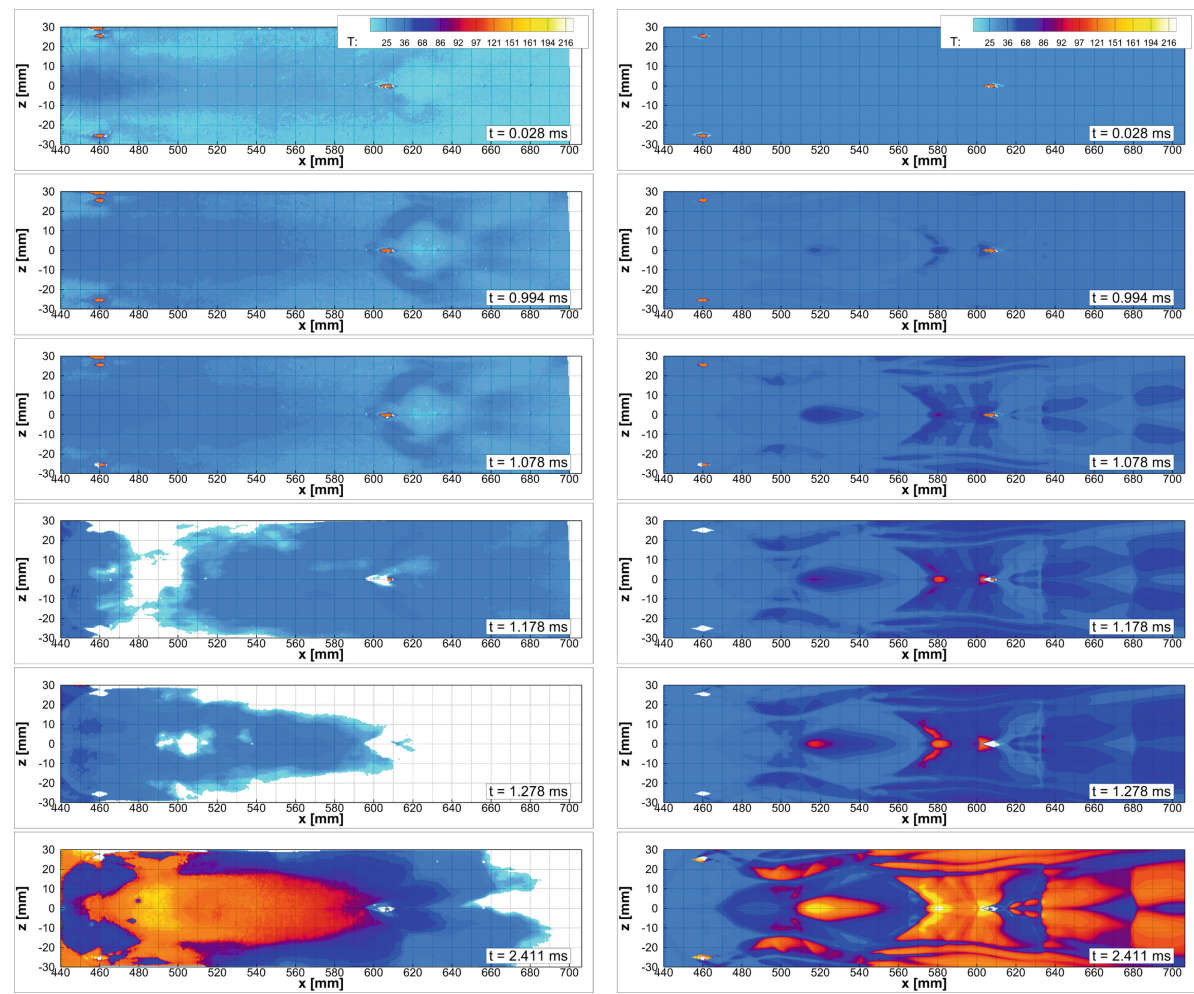

Fig. 5. Reconstructed temperature distribution at different times.

data is given in Fig. 5 for different time during the flow startup. The left column shows the experimental temperature evolution and corresponds to Fig. 4. We show the time $t=0.028 \mathrm{~ms}$ which is recorded prior to the arrival of the test gas expanding from the HEG nozzle at the model. It can be seen that the cooling of the wall due to the hydrogen, which is injected into the combustion chamber prior to this time instant, is picked up by the TSP. We then present a sequence $(t=1.111 \mathrm{~ms}, t=1.178 \mathrm{~ms}, t=1.278 \mathrm{~ms})$ showing that the hydrogen, which is already existing in the combustor, starts to burn at about $x=400 \mathrm{~mm}$ and is then blown downstream by the incoming flow. The main part of the combustion anchors at late times at a position of approximately $x=660 \mathrm{~mm}$. The right column of Fig. 5 shows the numerical calculation of the developing temperature. To obtain this time resolved numerical temperature data from the steady flow field calculation, the heat flux data from the solution has been transformed by the application of Eq. 2, here $t$ is set according to the experimental time. The numerical temperature distribution shows a temperature development for the assumption of a constant heat flux, which is not necessarily the case in the experiment, but is a quite fair assumption. Discussing the comparison, we can show that the overall agreement of the individual flow features is reasonable to 
some point, but certain specific features differ. The high local heating point on the symmetry axis of the combustor located at $x=520 \mathrm{~mm}$ in the numerical solution appears more upstream in the experimental data around $x=490 \mathrm{~mm}$. The characteristic footprint of the hydrogen-jet emerging from the two first stage semi strut injectors are better visible in the numerical data than in the experiment but the pattern is quite similar. The strong shock in the numerical data almost perpendicular to the flow direction at $x=490 \mathrm{~mm}$ is not visible in the experimental data and the high heating zone not as well. We have to be careful, with a more extended analysis, since the flow might not have reached a steady state at the early time, where the analysis has to stop. Usually we regard the flow to be stable after $t=3.00 \mathrm{~ms}$. A modified version of the acrylic glass cover will allow for extended experiments and these will be reported in the future.

\section{Summary, Conclusion and Outlook}

The feasibility of the application of TSP to internal flows has potential but comes with high demands. This experimental investigation has outlined several technical improvements for further experiments. A modified version of the acrylic glass cover will prevent the unwanted movement. To scope with the high heat loads during the experiment, the TSP has to be adapted with regard to the temperature sensitivity. The strong illumination of the hydrogen burning zones has to be suppressed. Emission spectroscopy to evaluate the emission wavelengths will be applied.

\section{References}

1. Hannemann, K., Martinez Schramm, J.: High enthalpy, high pressure short duration testing of hypersonic flows. In: Springer Handbook of Experimental Fluid Mechanics, pp. 1081-1125. Springer, Berlin Heidelberg (2007)

2. Deutsches Zentrum für Luft - und Raumfahrt (DLR). The high enthalpy shock tunnel Göttingen of the German aerospace center (DLR). J. large-scale Res. Facilit. (2018). https://doi.org/10.17815/jlsrf-4-168

3. Hannemann, K., Martinez Schramm, J., Laurence, S., Karl, S., Langener, T., Steelant, J.: Experimental and numerical analysis of the small scale LAPCAT II scramjet flow path in high enthalpy shock tunnel conditions (2014). In: Proceedings Space Propulsion 2014, SP2014-2969350

4. Karl, S., Martinez Schramm, J., Hannemann, K.: Post-test analysis of the LAPCAT-II subscale scramjet. In: HiSST: International Conference on High-Speed Vehicle Science Technology (2018)

5. Karl, S., Martinez Schramm, J., Hannemann, K.: Post-test analysis of the LAPCAT-II subscale scramjet. CEAS Space J. 12(3), 385-395 (2020). https:// doi.org/10.1007/s12567-020-00307-7

6. Martinez Schramm, J., Edzards, F., Hannemann, K.: Calibration of fast-response temperature sensitive paints for their application in hypersonic high enthalpy flows. In: New Results in Numerical and Experimental Fluid Mechanics XI, Notes on Numerical Fluid Mechanics and Multidisciplinary Design. Springer (2017). https:// doi.org/10.1007/978-3-319-64519-3_13 
7. J Martinez Schramm, S Karl, K Hannemann, and H Ozawa. Ultra-fast Temperature Sensitive Paint Shock Tunnel Heat Flux Measurements on the Intake of the LAPCAT II Small Scale Flight Experiment Configuration., (2018). HiSST: International Conference on High-Speed Vehicle Science Technology

8. Liu, T., Sullivan, J.P.: Pressure and Temperature Sensitive Paints. Springer-Verlag, Berlin Heidelberg (2005)

9. Martinez Schramm, J., Edzards, F., Hannemann, K.: Development of ultra-fast temperature sensitive paints for hypersonic high speed flows. In: 21st AIAA International Space Planes and Hypersonics Technologies Conference (2017). https:// doi.org/10.2514/6.2017-2211

10. Schultz, D.L., Jones, T.V.: Heat transfer measurements in short-duration hypersonic facilities. AGARD-AG-165 (1973)

11. Cook, W.J., Felderman, E.J.: Reduction of data from thin-film heat transfer gage: a concise numerical technique. AIAA J. 4(3), 561-562 (1966)

12. Martinez Schramm, J., Hannemann, K., Ozawa, H., Beck, W., Klein, C.: Development of temperature sensitive paints for the high enthalpy shock tunnel Göttingen HEG. In: 8th European Symposium on Aerothermodynamics for Space Vehicles (2015)

13. Steelant, J.: Sustained hypersonic flight in Europe: technology drivers for LAPCAT II. In: AIAA 2009-7240, 16th AIAA/DLR/DGLR International Space Planes and Hypersonic System Technologies Conference (2009) 